\title{
Involvement of the expression and redistribution of claudin-23 in pancreatic cancer cell dissociation
}

\author{
WEI WANG ${ }^{*}$, XIAODONG TAN ${ }^{*}$, LEI ZHOU, FENG GAO and XIANWEI DAI \\ Department of Pancreatic and Thyroidal Surgery, \\ China Medical University Shengjing Hospital, Shenyang 110004, P.R. China
}

Received April 20, 2010; Accepted June 28, 2010

DOI: $10.3892 / \mathrm{mmr} .2010 .334$

\begin{abstract}
Dissociation of pancreatic cancer cells from primary sites is the critical first step in tumour invasion and metastasis. Changes in the structure and function of tight junctions are reported to be correlated with carcinogenesis and tumour development. Using cDNA microarray analysis, we recently identified claudin-23 as a differentially expressed gene related to invasion-metastasis in highly (PC-1.0) and weakly (PC-1) invasive and metastatic pancreatic cancer cells. In this study, RT-PCR, Western blotting and immunocytochemistry were used to demonstrate the involvement of the expression and redistribution of claudin-23 in pancreatic cancer cell dissociation. Claudin-23 mRNA and protein were differentially expressed in PC-1.0 and PC-1 cells. Claudin-23 expression was induced in a PC-1.0 subclone expressing mitogen-activated protein kinase kinase (MEK)-1 shorthairpin RNA (shRNA) and claudin-23 was redistributed in a PC-1.0 subclone expressing MEK2 shRNA. Furthermore, these MEK2 shRNA-expressing PC-1.0 cells aggregated and formed island-like cell colonies. By contrast, the addition of dissociation factor-conditioned medium significantly reduced claudin-23 mRNA and protein expression in PC-1 cells. The present results indicate that claudin-23 is involved in the regulation of pancreatic cancer cell dissociation through changes in gene expression and intracellular localisation. In addition, claudin-23 expression is possibly correlated with the activation of the MEK signalling pathway during pancreatic cancer cell dissociation. Claudin-23 may thus serve as a new target for molecular therapies to prevent pancreatic cancer invasion and metastasis.
\end{abstract}

Correspondence to: Dr Xianwei Dai, Department of Pancreatic and Thyroidal Surgery, China Medical University Shengjing Hospital, No. 36, Sanhao Street, Heping, Shenyang 110004, P.R. China

E-mail: tanxd@hotmail.com

*Contributed equally

Key words: pancreatic cancer, cell dissociation, claudin-23, mitogen-activated protein kinase kinase

\section{Introduction}

Pancreatic cancer is one of the most malignant tumours and presents a high frequency of invasion and metastasis. Dissociation of pancreatic cancer cells from primary sites is the critical first step in tumour invasion and metastasis (1-3); however, the molecular mechanisms by which this detachment occurs remain unclear. Clarifying the molecular mechanisms of cell dissociation may contribute to the elucidation of the mechanisms of pancreatic cancer invasion and metastasis and to the development of new clinical therapies that may improve the prognosis of pancreatic cancer patients.

In our previous study, dissociation factor (DF) was isolated from the culture medium of highly invasive and metastatic PC-1.0 pancreatic cancer cells, and was shown to induce the dissociation of weakly invasive and metastatic pancreatic cancer cells (PC-1) $(4,5)$. In further investigations, DF was found to activate the mitogen-activated protein kinase kinase (MEK)/extracellular signal-regulated kinase (ERK) pathway, which was identified as a pivotal signalling pathway related to cell dissociation in PC-1.0 and PC-1 cells (6-8).

In addition, we recently used cDNA microarray analysis to identify claudin- 23 as a differentially expressed gene related to invasion-metastasis in PC-1.0 and PC-1 cells (9). Tight junctions (TJs) are intercellular junctional complexes located at the apical end of the lateral membranous surface of polarised cells. Three groups of macromolecules are considered integral components of TJs: occludins, claudins and junctional adhesion molecules $(10,11)$. Claudins are the key proteins for the sealing of the extracellular space (12-15). The claudin family consists of 24 members. Different claudins are found at the TJs of diverse epithelial cells (16), and this accounts for the observed differences in permeability and electrical resistance of various epithelia $(10,13,15)$. Previous studies have shown that claudins are deregulated in a variety of malignancies; for example, the up-regulation of claudin- 3 and -4 accompanies the progression of endometrial carcinoma $(17,18)$, reduced expression of claudin-1 correlates with poorer differentiation, disease recurrence and poor survival in stage II colonic cancer (19), claudin-7 expression is reduced in mammary (20), oesophageal (21) and head and neck carcinomas (22), and claudin- 4 overexpression decreases the invasiveness and metastatic potential of pancreatic cancer cells (23). Claudin-23 is a recently identified member of the claudin gene family 
that is expressed in germinal centre B cells, skin, placenta, stomach and colon tumours $(24,25)$. A recent report demonstrated that claudin-23 may be a candidate tumour suppressor gene in intestinal-type gastric cancer (24); however, the role of claudin-23 in tumour invasion and metastasis, and especially in cell dissociation during pancreatic cancer, remains unclear.

To clarify the involvement of claudin-23 in pancreatic cancer cell dissociation, we analysed the correlation between changes in claudin-23 expression and intracellular distribution and pancreatic cancer cell dissociation.

\section{Materials and methods}

Cell lines and cell culture. Two hamster pancreatic cancer cell lines were used: the weakly invasive and rarely metastatic cell line PC-1 and the highly invasive and metastatic cell line PC-1.0. The PC-1 cell line was established from pancreatic ductal/ductular adenocarcinomas induced by BOP in a Syrian golden hamster (26). The PC-1.0 cell line was established from a subcutaneous tumour produced after inoculation with PC-1 cells (27). These two cell lines exhibited different growth morphology in vitro. The PC-1 cells formed island-like cell colonies, whereas PC-1.0 cells mainly grew as single cells (28). In addition, PC-1.0 subclones expressing MEK1 or MEK2 short-hairpin RNA (shRNA) were used to specifically silence the expression of MEK1 or MEK2 in the highly invasive and metastatic $\mathrm{PC}-1.0$ pancreatic cancer cell line (29).

All cell lines were incubated in RPMI-1640 (Gibco BRL, Grand Island, NY, USA) supplemented with $10 \%$ foetal bovine serum (FBS; Bioserum, Victoria, Australia), $100 \mathrm{U} /$ $\mathrm{ml}$ penicillin $\mathrm{G}$ and $100 \mu \mathrm{g} / \mathrm{ml}$ streptomycin at $37^{\circ} \mathrm{C}$ in a humidified atmosphere of $5 \% \mathrm{CO}_{2}$ and $95 \%$ air. The cells were serum-starved overnight before the experiments.

Antibodies. Rabbit polyclonal antibodies raised against amino acid sequences of human claudin-23 (Abcam Inc., Cambridge, UK) and $\beta$-actin (Santa Cruz Biotechnology, Santa Cruz, CA, USA) were used as primary antibodies. Horseradish peroxidase-conjugated and FITC-labelled fluorescent antibodies (Santa Cruz Biotechnology) were used as secondary antibodies for Western blotting and immunofluorescence staining, respectively.

Reverse transcriptase-polymerase chain reaction (RT-PCR). Total RNA was extracted from each culture with TRIzol reagent (Life Technologies, Inc., Gaithersburg, MD, USA). RT-PCR was performed with the Superscript II One-Step RT-PCR System (Life Technologies, Inc.) in a PTC-200 Peltier Thermal Cycler (MJ Research, Inc., USA). The specific primers used for amplification were as follows: 5'- ACGGCAGGGAGAAGACGA (forward) and 5'-AGCGACGAAGAGCACGAC (reverse) for claudin-23 (457 bp); 5'-GTGGGGCGCCCCAGGCACCA (forward) and 5'-CTCCTTAAGTCACGCACGATTCC (reverse) for $\beta$-actin (664 bp). The PCR protocol was as follows: $95^{\circ} \mathrm{C}$ for $5 \mathrm{~min}$ followed by 30 cycles of $94^{\circ} \mathrm{C}$ for $30 \mathrm{sec}, 58^{\circ} \mathrm{C}$ for $30 \mathrm{sec}$ and $72^{\circ} \mathrm{C}$ for $1 \mathrm{~min}$, and a final extension step at $72^{\circ} \mathrm{C}$ for $7 \mathrm{~min}$. PCR products were electrophoresed and visualised on $1.5 \%$ agarose gels containing ethidium bromide.
Preparation of cell lysates. Cells were grown in 90-mm dishes containing $10 \mathrm{ml}$ of RPMI-1640 supplemented with $10 \%$ FBS.

To evaluate the expression of claudin-23 protein and the relationship between the expression of claudin-23 and the activation of the MEK1/2 signalling pathway during pancreatic cancer cell dissociation, the conditioned medium (CM) of PC-1.0 cells (DF-CM) was applied to PC-1 cells for activation studies (30). PC-1 cells were incubated with $40 \%$ DF-CM for $36 \mathrm{~h}$. The PC-1.0 subclones expressing MEK1 shRNA or MEK2 shRNA were used for inhibition studies.

The cells were lysed in $1 \mathrm{ml}$ ice-cold RIPA buffer $(50 \mathrm{mM}$ Tris, $150 \mathrm{mM} \mathrm{NaCl}, 1 \% \mathrm{NP}-40,0.5 \%$ sodium deoxycholate, $0.1 \%$ SDS, $\mathrm{pH} 7.5$ ) containing $1 \mathrm{mM}$ phenylmethylsulfonyl fluoride, $1 \mathrm{mg} / \mathrm{ml}$ leupeptin and $1 \mathrm{mg} / \mathrm{ml}$ aprotinin on ice for $15 \mathrm{~min}$. After centrifugation $(5 \mathrm{~min}$ at $5,000 \mathrm{rpm})$ at $4^{\circ} \mathrm{C}$, the cell lysate supernatants were collected and stored at $-80^{\circ} \mathrm{C}$. $\beta$-actin was used as an internal control.

Western blot analysis. Western blotting was performed as described previously (31). In brief, samples containing equivalent total protein $(20 \mu \mathrm{g})$ were separated in a $5 \%$ polyacrylamide slab gel and transferred to polyvinylidene fluoride (PVDF) membranes (BIO-RAD, Anaheim, CA, USA). The membranes were then incubated with primary antibody diluted in $0.1 \%$ Tween-20/PBS overnight at $4^{\circ} \mathrm{C}$, followed by horseradish peroxidase-conjugated secondary antibody diluted 1:5,000 in 0.1\% Tween-20/PBS. Enhanced chemiluminescence (Santa Cruz Biotechnology) was used to detect the signals developed on Kodak scientific imaging film (Eastman Kodak Company, Rochester, NY, USA).

Immunofluorescent staining and fluorescence intensity analysis. Cells were seeded on chamber slides and incubated for $36 \mathrm{~h}$ before experiments. To evaluate the redistribution of claudin-23 protein and the relationship between the expression of claudin-23 and pancreatic cancer cell dissociation, DF-CM was applied to PC-1 cells. Cells were treated as described in Preparation of cell lysates. In addition, PC-1.0 subclones expressing MEK1 shRNA or MEK2 shRNA were used.

All of the cells mentioned above were fixed with $0.5 \%$ paraformaldehyde for $10 \mathrm{~min}$ at room temperature. Cells were blocked with $10 \%$ normal goat serum for $30 \mathrm{~min}$. Slides were then incubated with polyclonal anti-claudin-23 antibody (1:200 dilution in $1 \%$ bovine albumin in PBS) at $4^{\circ} \mathrm{C}$ overnight, followed by incubation with FITC-labelled secondary antibody for $2 \mathrm{~h}$ at room temperature. The slides were washed three times with PBS between each step. After mounting, immunofluorescence images were captured with a confocal laser scanning biological microscope (FV500-IX; Olympus, Japan). The control slides were prepared as follows: i) slides were processed without a primary antibody; ii) normal rabbit serum and non-specific rabbit IgG were used instead of a polyclonal anti-claudin-23 antibody.

Finally, 10 images each of the nucleus, cytoplasm, membrane and whole cell were selected at random, and claudin-23 protein expression was measured in each image by analysing fluorescence intensity (FI) with Fluoview 500 software (version 3.3; Olympus). 


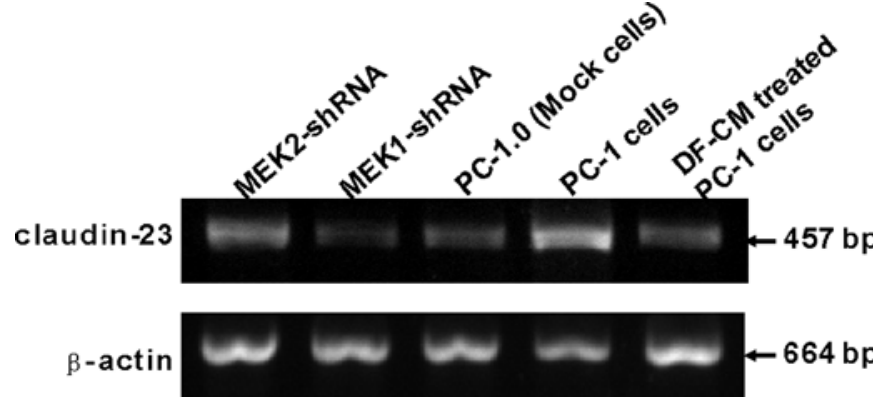

Figure 1. Changes in claudin-23 mRNA expression in highly (PC-1.0) and weakly (PC-1) invasive and metastatic pancreatic cancer cell lines.

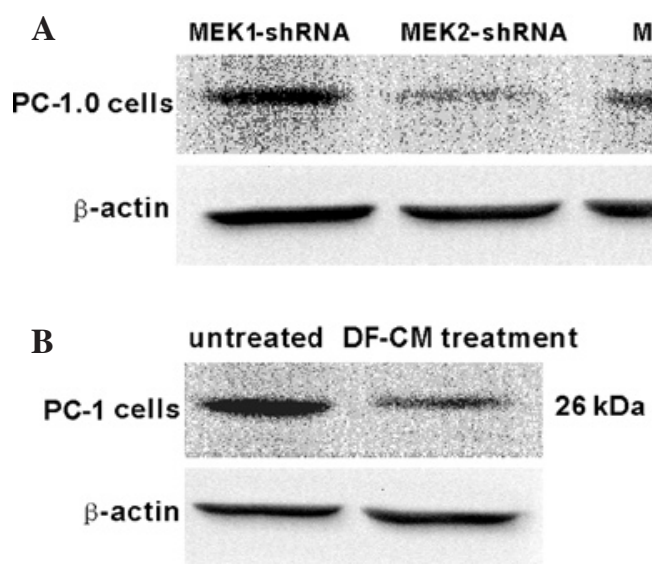

Figure 2. Western blotting of claudin-23 protein in highly (PC-1.0) and weakly (PC-1) invasive and metastatic pancreatic cancer cells. The expression of claudin-23 protein was weak in control PC-1.0 cells (A). The expression of claudin-23 protein was induced in PC-1.0 subclones expressing MEK1 shRNA, but not in PC-1.0 subclones expressing MEK2 shRNA (A). By contrast, claudin-23 expression was strong in untreated PC-1 cells (B). A significant reduction in claudin-23 expression was observed in PC-1 cells after a $36-\mathrm{h}$ treatment with DF-CM (B). The molecular weight marker is indicated.

Statistical analysis. The average FI of claudin- 23 in 10 images of nucleus, cytoplasm, membrane and whole cells in various experimental groups was examined, and the statistical significance of differences between the groups was determined by an unpaired Student's t-test performed using the Stat View computerised program (SAS Institute, Inc., Cary, NC, USA). Probability values of $<0.05$ were considered significant.

\section{Results}

Changes in claudin-23 mRNA expression in pancreatic cancer cells. Claudin- 23 mRNA was constitutively expressed in the weakly invasive and metastatic PC-1 pancreatic cancer cells. Moreover, the constitutive expression of claudin- 23 mRNA in these cells was significantly inhibited after 24-h treatment with DF-CM (Fig. 1).

Conversely, the expression of claudin-23 mRNA was much lower in the highly invasive and metastatic PC-1.0 pancreatic cancer cells. Claudin-23 mRNA expression was induced in PC-1.0 subclones that expressed MEK1 shRNA, but not in those that expressed MEK2 shRNA (Fig. 1).
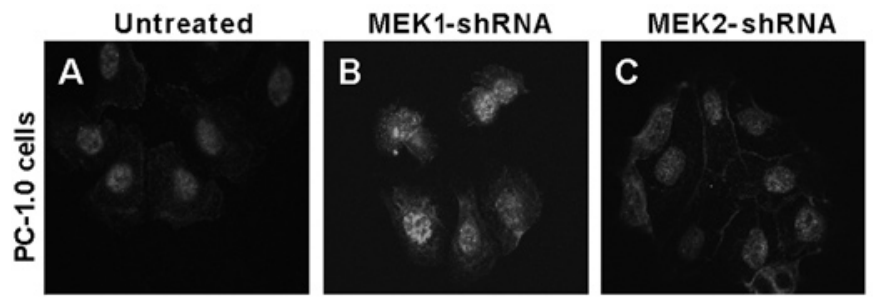

Untreated

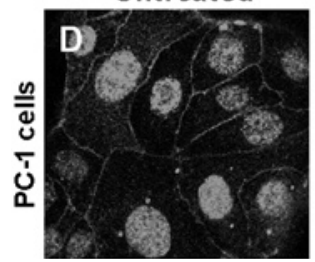

DF-CM treatment

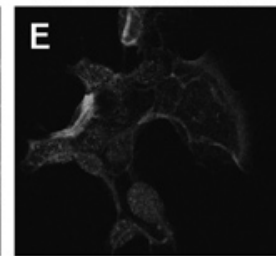

Figure 3. Rearrangement of claudin-23 localisation in highly (PC-1.0) and weakly (PC-1) invasive and metastatic pancreatic cancer cells. Claudin-23 protein was mainly localised to the nucleus in the control PC-1.0 cells (A); however, markedly enhanced localisation of claudin-23 protein was found in the nucleus of PC-1.0 subclones expressing MEK1 shRNA (B). The localisation of claudin-23 protein at the sites of cell-cell contact was significantly induced in PC-1.0 subclones expressing MEK2 shRNA (C). By contrast, untreated PC-1 cells showed localisation of claudin-23 to the sites of cell-cell junctions and to the nucleus (D). The localisation of claudin-23 protein to the sites of cell-cell junctions and to the nucleus was disrupted by a 36-h DF-CM treatment (E). Immunofluorescence staining: original magnification, $\mathrm{x} 400$.

Western blotting of claudin-23 protein in pancreatic cancer cells. Western blotting data showed that claudin-23 protein was expressed at much lower levels in the highly invasive and metastatic PC-1.0 pancreatic cancer cells (Fig. 2A). Similar to the changes observed in mRNA expression, claudin- 23 protein expression was induced in PC-1.0 subclones that expressed MEK1 shRNA, but not in those that expressed MEK2 shRNA (Fig. 2A). Claudin-23 protein was strongly expressed in the weakly invasive and metastatic PC-1 pancreatic cancer cells (Fig. 2B); however, DF-CM treatment resulted in the suppression of the claudin-23 protein expression in these cells (Fig. 2B).

Redistribution of claudin-23 protein in pancreatic cancer cells. In the control PC-1.0 cells (Fig. 3A), claudin-23 protein was found mainly in the nucleus, while faint expression was detected at the cellular periphery and in the cytoplasm; however, there was a marked increase in claudin- 23 in the nucleus of PC-1.0 subclones expressing MEK1 shRNA (Fig. 3B). By contrast, claudin-23 expression was induced at the sites of cell-cell contact in PC-1.0 subclones expressing MEK2 shRNA (Fig. 3C).

In the untreated PC-1 cells (Fig. 3D), claudin-23 protein was exclusively localised to the nucleus and the peripheral sites of cell-cell contact, and only faint levels of claudin-23 were detected in the cytoplasm. The peripheral distribution of claudin-23 at the sites of cell-cell contact in PC-1 cells was disrupted and became discontinuous after treatment with DF-CM for $36 \mathrm{~h}$ (Fig. 3E). Furthermore, the intranuclear localisation of claudin-23 was decreased in DF-CM-treated PC-1 cells.

Fluorescence intensity of claudin-23 expression in pancreatic cancer cells. The results of FI analysis of claudin-23 
A

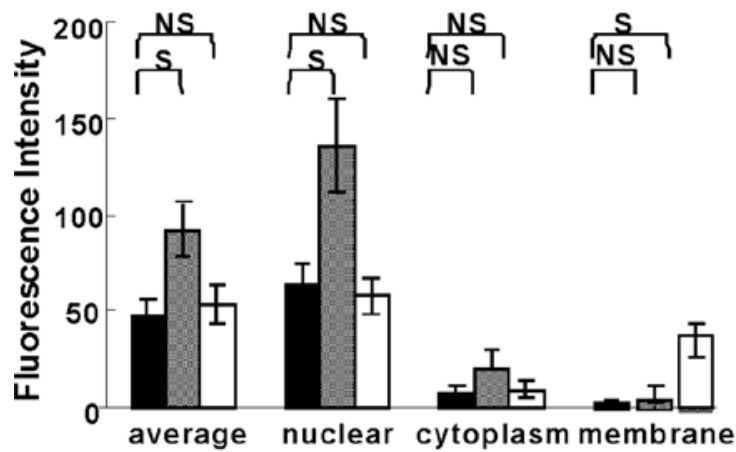

B

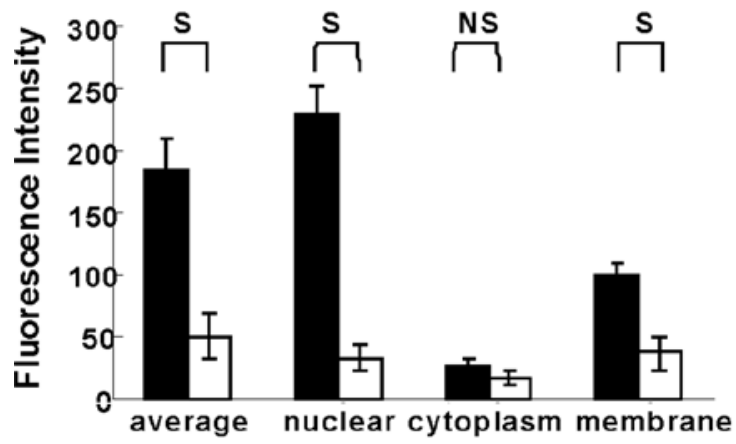

Figure 4. Fluorescence intensity (FI) of claudin-23 expression in highly (PC-1.0) and weakly (PC-1) invasive and metastatic pancreatic cancer cells. (A) FI values of claudin-23 expression in PC-1.0 cells. Black bar, control PC-1.0 cells; dotted bar, PC-1.0 subclones expressing MEK1 shRNA; white bar, PC-1.0 subclones expressing MEK2 shRNA; S, significant; NS, not significant. (B) FI values of claudin-23 expression in PC-1 cells. Black bar, untreated PC-1 cells; white bar, DF-CM-treated PC-1 cells; S, significant; NS, not significant.

expression in PC-1.0 cells and PC-1 cells are shown in Fig. 4A and $\mathrm{B}$, respectively.

The average whole cell expression of claudin-23 was low in control PC-1.0 cells (average FI=46.82 \pm 8.91 ; Fig. 4A). The claudin-23 protein was mainly localised to the nucleus in these cells $(\mathrm{FI}=63.78 \pm 10.01)$ and almost no fluorescence was detected in the cytoplasm (FI=6.86 \pm 4.21$)$ or at the cellular periphery (FI=4.05 \pm 2.06 ). In $\mathrm{PC}-1.0$ subclones expressing MEK1 shRNA, the nuclear and average whole cell expression levels of claudin-23 were significantly increased (average $\mathrm{FI}=135.06 \pm 24.45$ and $91.52 \pm 13.27$, respectively, $\mathrm{P}<0.05$ ). As noted previously, there was no obvious claudin-23 localisation in the cytoplasm and at the sites of cell-cell contact ( $\mathrm{FI}=19.88 \pm 9.15$ and $5.71 \pm 3.05, \mathrm{P}>0.05)$. In addition, in PC-1.0 subclones expressing MEK2 shRNA, the expression of claudin-23 at the sites of cell-cell contact was significantly induced $(\mathrm{FI}=36.54 \pm 8.67, \mathrm{P}<0.05)$, although there was no significant change in the amount of claudin-23 in the nucleus ( $\mathrm{FI}=57.66 \pm 8.87, \mathrm{P}>0.05)$, cytoplasm $(\mathrm{FI}=8.67 \pm 4.23, \mathrm{P}>0.05)$ or throughout the entire cell $(\mathrm{FI}=53.47 \pm 10.16, \mathrm{P}>0.05)$.

The average whole cell FI of claudin-23 expression was $183.56 \pm 27.68$ in untreated PC-1 cells (Fig. 4B). We observed strong claudin- 23 expression in the nucleus $(\mathrm{FI}=229.80 \pm 23.05)$ and at sites of cell-cell contact (FI=98.53 \pm 11.34 ), while claudin-23 expression in the cytoplasm was weak $(\mathrm{FI}=26.42 \pm 6.19)$. After treatment with DF-CM for $36 \mathrm{~h}$, the expression of claudin- 23
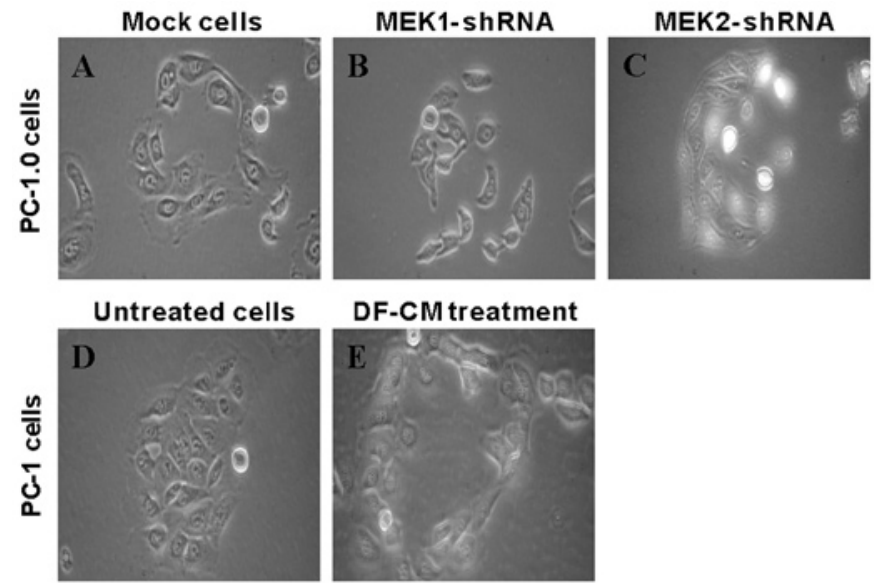

Figure 5. Morphological changes in highly (PC-1.0) and weakly (PC-1) invasive and metastatic pancreatic cancer cells. Untreated PC-1.0 cells (A) aggregated and formed island-like cell colonies in PC-1.0 subclones expressing MEK2 shRNA (C), although no obvious morphological changes were observed in PC-1.0 subclones expressing MEK1 shRNA (B). The island-like cell colonies of untreated PC-1 cells (D) were dissociated by a 36-h DF-CM treatment (E). Papanicolaou's staining: original magnification, $\mathrm{x} 400$

in the nucleus $(\mathrm{FI}=32.64 \pm 11.26, \mathrm{P}<0.05)$, at sites of cell-cell contact $(37.22 \pm 13.57, \mathrm{P}<0.05)$ and throughout the entire cell (average FI $=51.04 \pm 18.27, \mathrm{P}<0.05$ ) was significantly reduced compared to that in untreated cells, although claudin-23 expression in the cytoplasm ( $\mathrm{FI}=17.36 \pm 5.63, \mathrm{P}>0.05)$ was not significantly changed.

Changes in cell dissociation status in pancreatic cancer cells. Light microscopic images showed that untreated PC-1.0 cells grew as single cells (Fig. 5A). The PC-1.0 subclones expressing MEK1 shRNA showed no significant morphological changes compared to control PC-1.0 cells (Fig. 5B). Notably, PC-1.0 subclones expressing MEK2 shRNA aggregated and formed island-like cell colonies (Fig. 5C), similar to those observed in untreated PC-1 cells (Fig. 5D). After a 36-h incubation with DF-CM, the island-like colonies of PC-1 cells became dissociated and the cells exhibited morphological changes, becoming elongated with a high frequency of pseudopodia formation (Fig. 5E), similar to the morphology of the PC-1.0 cells.

\section{Discussion}

Various cancers (i.e., ovarian, colon and cervical cancer) have been reported to be associated with elevated $\mathrm{TJ}$ protein expression levels or protein dislocation to the cytoplasm (32-34). These reports emphasise the complex and diverse actions of TJ proteins in cancer progression.

In our recent study, the TJ protein claudin- 23 was identified as an invasion-metastasis-related factor by comparing highly invasive and metastatic PC-1.0 pancreatic cancer cells with weakly invasive and metastatic PC-1 pancreatic cancer cells via cDNA microarrays (9).

In our previous studies, we found that DF induces the activation of the MEK/ERK signal transduction pathway and 
pancreatic cancer cell dissociation $(7,8)$. Moreover, inhibition of expression and activation of components of the MEK/ ERK signal transduction pathway and changes in cell dissociation and proliferation were observed in PC-1.0 subclones expressing MEK1 shRNA and MEK2 shRNA (29).

In the present study, we found that claudin-23 mRNA was differentially expressed in highly (PC-1.0) and weakly (PC-1) invasive and metastatic pancreatic cancer cells. Moreover, DF-CM was found to decrease claudin-23 mRNA and protein expression. Interestingly, claudin-23 mRNA and protein expression were found to be induced in PC-1.0 subclones expressing MEK1 shRNA. Moreover, claudin-23 protein redistribution and cell morphological changes were also induced in PC-1.0 subclones expressing MEK2 shRNA. These results demonstrate that claudin-23 may be involved in the regulation of cell dissociation, which is the first pivotal step in pancreatic cancer cell invasion-metastasis. Furthermore, gene expression and intracellular distribution of claudin-23 may be regulated by the MEK/ERK signal transduction pathway. The present results also suggest that the expression and intracellular distribution of claudin-23 protein during pancreatic cancer cell dissociation are possibly regulated by the MEK1 and MEK2 signal transduction subpathways, respectively.

Notably, our results show that DF changes the intracellular localisation of claudin-23 protein. Moreover, the intracellular localisation of claudin-23 protein is closely correlated with the cell dissociation status of pancreatic cancer cells. These results reveal that proper localisation of claudin-23 to sites of cell-cell contact may be important for sustaining the stability of TJs and preventing cell dissociation and subsequent pancreatic cancer cell invasion-metastasis. Furthermore, changes in the intracellular localisation of claudin-23 may be involved in the regulation of cell dissociation and invasion-metastasis.

In our present study, the nuclear localisation observed in immunofluorescent images indicated that claudin-23 may serve not only as a TJ transmembrane protein, but also as an intracellular signalling molecule. Since the molecular weight of claudin-23 is $26 \mathrm{kDa}$, and since molecules less than $45 \mathrm{kDa}$ are theoretically capable of passing through the nuclear membrane, the observations in this study provide evidence to support the possibility that claudin-23 also serves as a signalling molecule by diffusing or being actively transported to the nucleus from the site of cell-cell adhesion, while the structure of the TJ is disrupted.

In addition, certain members of the claudin family can be phosphorylated, and changes in claudin phosphorylation may regulate its intracellular localisation (35-37). Since the translocation of claudin-23 protein between the nucleus, cytoplasm and cell membrane was found to correlate with the activation (phosphorylation) of MEK2 signalling molecules in this study, the change in the phosphorylation of claudin-23 may be regulated by the MEK2 signal transduction pathway during pancreatic cancer cell dissociation. Further investigation is required to determine the role of claudin- 23 phosphorylation in the regulation of cell dissociation in pancreatic cancer.

In summary, claudin-23 is involved in the regulation of pancreatic cancer cell dissociation through changes in expression and intracellular localisation. Claudin-23 may serve as a new target for molecular therapies that prevent the invasion and metastasis of pancreatic cancer.

\section{Acknowledgements}

The study was sponsored by a grant-in-aid from the National Nature Science Foundation of China (no. 30973501) and the Scientific Research Foundation for the Returned Overseas Chinese Scholars, State Education Ministry (no. 2007-1108). We would like to acknowledge Professor Hideo Baba for the kind gift of the PC-1 and PC-1.0 cell lines.

\section{References}

1. Torimura T, Ueno T, Kin M, et al: Autocrine motility factor enhances hepatoma cell invasion across the basement membrane through activation of $\beta 1$ integrins. Hepatology 34: 62-71, 2001.

2. Fincham VJ, James M, Frame MC, et al: Active ERK/MAP kinase is targeted to newly forming cell-matrix adhesions by integrin engagement and v-Src. EMBO J 19: 2911-2923, 2000.

3. Rescan C, Coutant A, Talarmin H, et al: Mechanism in the sequential control of cell morphology and $S$ phase entry by epidermal growth factor involves distinct MEK/ERK activations. Mol Biol Cell 12: 725-738, 2001.

4. Hirota M, Egami H, Corra S, et al: Production of scatter factorlike activity by a nitrosamine-induced pancreatic cancer cell line. Carcinogenesis 14: 259-264, 1993.

5. Kurizaki T, Egami H, Hirota M, et al: Characterization of cancer cell dissociation factor in a highly invasive pancreatic cancer cell line. Cancer 75: 1554-1561, 1995.

6. Ishikawa S, Egami H, Kurizaki T, et al: Analysis of the factor related to the signal transduction pathway of invasion and metastasis in pancreatic cancer. J Exp Clin Cancer Res 22: 299-306, 2003.

7. Tan X, Egami H, Kamohara H, et al: Involvement of the mitogen-activated protein kinase kinase 2 in the induction of cell dissociation in pancreatic cancer. Int J Oncol 24: 65-73, 2004.

8. Tan X, Egami H, Ishikawa S, et al: Relationship between the expression of extracellular signal-regulated kinase $1 / 2$ and the dissociation of pancreatic cancer cells: Involvement of ERK1/2 in the dissociation status of cancer cells. Int J Oncol 24: 815-820, 2004.

9. Tan X, Zhou L, Wang W, et al: Genomic analysis of invasionmetastasis related factors in pancreatic cancer cells. Exp Ther Med 1: 211-216, 2010.

10. Gonzalez-Matrisal L, Bentazos A, Nava P, et al: Tight junction proteins. Prog Bio Mol Biol 18: 1-44, 2003.

11. Anderson JM and van Itallie CM: Tight junctions and the molecular basis for regulation of paracellular permeability. Am J Physiol 227: G467-G475, 1995.

12. Furuse M, Fujita K and Hiiragi T: Claudin-1 and-2: novel integral membrane proteins localizing at tight junctions with no sequence similarity to occludin. J Cell Biol 141: 1539-1550, 1998.

13. Mitic LL, Itallie CM and Anderson JM: Molecular physiology and pathophysiology of tight junctions. I. Tight junction structure and function: lessons from mutant animals and proteins. Am J Physiol Gastrointest Liver Physiol 279: G250-G254, 2000.

14. Tsukita S and Furuse M: Pores in the wall: claudins constitute tight junction strands containing aqueous pores. J Cell Biol 149: 13-16, 2000.

15. Rahner C, Mitic LL and Anderson JM: Heterogeneity in expression and subcellular localization of claudins 2,3,4 and 5 in the rat liver, pancreas and gut. Gastroenterology 120: 411-422, 2001.

16. Pan XY, Wang B, Che YC, et al: Expression of claudin-3 and 4 in normal, hyperplastic, and malignant endometrial tissue. Int J Gynecol Cancer 17: 233-241, 2007.

17. Tobioka H, Isomura H, Kokai Y, et al: Occludin expression decreases with the progression of human endometrial carcinoma. Hum Pathol 35: 159-164, 2004.

18. Resnick MB, Konkin T, Routhier J, et al: Claudin-1 is a strong prognostic indicator in stage II colonic cancer: a tissue microarray study. Mod Pathol 18: 511-518, 2005.

19. Kominsky SL, Agrani P, Korz D, et al: Loss of tight junction protein claudin-7 correlates with histological grade in both ductal carcinoma in situ and invasive ductal carcinoma of the breast. Oncogene 22: 2021-2033, 2003.

20. Usami Y, Chiba H, Nakayama F, et al: Reduced expression of claudin-7 correlates with invasion and metastasis in squamous cell carcinoma of the esophagus. Hum Pathol 37: 569-577, 2006. 
21. Swisshelm K, Macek R and Kubbies M: Role of claudins in carcinogenesis. Adv Drug Deliv Rev 57: 919-928, 2005.

22. Michl P, Barth C, Buchholz M, et al: Claudin-4 expression decreases invasiveness and metastatic potential of pancreatic cancer. Cancer Res 63: 6265-6271, 2003.

23. Hough CD, Sherman-Baust CS, Pizer ES, et al: Largescale serial analysis of gene expression reveals genes differentially expressed in ovarian cancer. Cancer Res 60: 6281-6287, 2000.

24. Katoh $M$ and Katoh M: CLDN23 gene, frequently downregulated in intestinal-type gastric cancer, is a novel member of CLAUDIN gene family. Int J Mol Med 11: 683-689, 2003.

25. Michlig S, Damak S and Coutre JL: Claudin-based permeability barriers in taste buds. J Comp Neurol 502: 1003-1011, 2007.

26. Egami H, Takiyama Y, Cano M, et al: Establishment of hamster pancreatic ductal carcinoma cell line (PC-1) producing blood group-related antigens. Carcinogenesis 10: 861-869, 1989.

27. Egami H, Tomioka T, Tempero M, Kay D and Pour PM: Development of intrapancreatic transplantable model of pancreatic duct adenocarcinoma in Syrian golden hamsters. Am J Pathol 138: 557-561, 1991.

28. Pour PM, Egami H and Takiyama Y: Patterns of growth and metastases of induced pancreatic cancer in relation to the prognosis and its clinical implications. Gastroenterology 100 : 529-536, 1991

29. Zhou L, Tan X, Kamohara H, et al: MEK1 and MEK2 isoforms regulate distinct functions in pancreatic cancer cells. Oncol Rep 24: $251-255,2010$.
30. Tan X, Egami H, Nozawa F, Abe M and Baba H: Analysis of the invasion-metastasis mechanism in pancreatic cancer: Involvement of palsmin(ogen) cascade proteins in the invasion of pancreatic cancer cells. Int J Oncol 28: 369-374, 2006.

31. Tan X, Egami H, Ishikawa S, et al: Involvement of matrix metalloproteinase-7 in invasion-metastasis through induction of cell dissociation in pancreatic cancer. Int J Oncol 26: 1283-1289, 2005.

32. Rangel LB, Agrawal R, D'Souza T, et al: Tight junction proteins claudin 3 and claudin 4 are frequently overexpressed in ovarian cancer but not in ovarian cystadenomas. Clin Cancer Res 9: 2567-2575, 2003.

33. Lee JW, Lee SJ, Seo J, et al: Increased expressions of claudin-1 and claudin-7 during the progression of cervical neoplasia. Gynecol Oncol 97: 53-59, 2005.

34. Hewitt KJ, Agarwal R and Morin PJ: The claudin gene family: expression in normal and neoplastic tissues. BMC Cancer 6: 186, 2006.

35. D'Souza T, Agarwal R and Morin PJ: Phosphorylation of claudin-3 at threonine 192 by cAMP-dependent protein kinase regulates tight junction barrier function in ovarian cancer cells. J Biol Chem 280: 26233-26240, 2005.

36. Stamatovic SM, Dimitrijevic OB, Kepp RF, et al: Protein kinase $\mathrm{C} \alpha$-RhoA cross talk in CCL2-induced alterations in brain endothelial permeability. J Biol Chem 281: 8379-8388, 2006.

37. Yamauchi K, Rai T, Kobayashi K, et al: Disease-causing mutant WNK4 increases paracellular chloride permeability and phosphorylates claudins. Proc Natl Acad Sci USA 101: 4690-4694, 2004. 